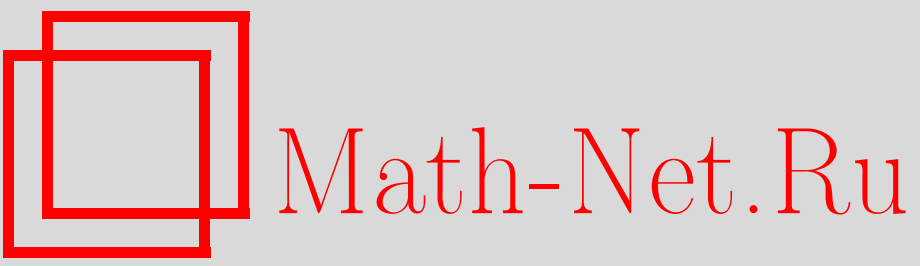

Р. Балакришнан, С. Муругеш, Кинематика трех подвижных пространственных кривых, связанных с нелинейным уравнением Шредингера, ТМ $\Phi, 2002$, том 133, номер 3, 341-352

DOI: https://doi.org/10.4213/tmf402

Использование Общероссийского математического портала Math-Net.Ru подразумевает, что вы прочитали и согласны с пользовательским соглашением

http://www.mathnet.ru/rus/agreement

Параметры загрузки:

IP : 52.205.19.152

26 апреля 2023 г., 12:12:37

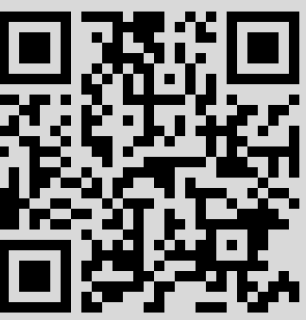




\author{
ТЕОРЕТИЧЕСКАЯ \\ И МАТЕМАТИЧЕСКАЯ \\ ФИЗИКА \\ Том 133, № 3 \\ декабрь, 2002
}

(C) 2002 г.

Р. Балакришнан*, С. Муругеш*

\title{
КИНЕМАТИКА ТРЕХ ПОДВИЖНЫХ ПРОСТРАНСТВЕННЫХ КРИВЫХ, СВЯЗАННЫХ С НЕЛИНЕЙНЫМ УРАВНЕНИЕМ ШРЕДИНГЕРА
}

На основании общего описания подвижной кривой ранее был представлен единый формализм, показывающий, что три различные эволюции пространственных кривых можно отождествить с данным интегрируемым уравнением. Применение этого формализма к нелинейному уравнению Шредингера (НШ) позволило найти три набора связанных уравнений для эволюции кривизны и кручения, по одному набору для каждой подвижной кривой. Первый набор имеет вид обычных уравнений Да Риоса-Бетчова. Хорошо известно, что скорость в каждой точке кривой из этого набора является локальным выражением по переменным кривой. Показано, что скорости двух других кривых являются нелокальными выражениями. Каждая из трех кривых снабжается соответствующим бесконечным набором геометрических связей. Эти подвижные пространственные кривые найдены с использованием их связи с интегрируемым уравнением Ландау-Лифшица. Приводятся три эволюционирующие кривые, соответствующие обертывающему солитонному решению уравнения НШ, и сравнивается их поведение.

Ключевые слова: солитоны, эволюция пространственных кривых, вихревые нити, нелинейное уравнение Шредингера.

\section{1. ВВЕДЕНИЕ}

Исследование возможных связей между внутренней кинематикой пространственных кривых (см., например, [1]) и интегрируемыми уравнениями, допускающими солитонные решения (см., например, [2]), заслуживает внимания из-за широкого разнообразия применений подвижных кривых, таких как движение вихревых нитей в жидкостях [3], динамика континуальных спиновых цепочек [4], спиральные волны в химических реакциях (см. [5] и приведенную там литературу), сверхтекучесть [6], динамика раздела сред [7] и т. д. Такая связь была впервые предложена в пионерских работах Хасимото [3], описываюших движение вихревых нитей в жидкости. В классической дифференциальной геометрии пространственная кривая, допускающая вложение в трехмерное прос-

*The Institute of Mathematical Sciences, Chennai, India. E-mail: radha@imsc.ernet.in, mgesh@imsc.ernet.in 
транство, описывается с использованием следующих уравнений Френе-Серре [1] для ортонормальной тройки единичных векторов, составленной из ее касательной $\mathbf{t}$, нормали $\mathbf{n}$ и бинормали $\mathbf{b}$ :

$$
\mathbf{t}_{s}=K \mathbf{n}, \quad \mathbf{n}_{s}=-K \mathbf{t}+\tau \mathbf{b}, \quad \mathbf{b}_{s}=-\tau \mathbf{n}
$$

Здесь $s$ обозначает длину дуги, параметры $K$ и $\tau$ представляют собой кривизну и кручение пространственной кривой. Для подвижной кривой они являются функциями как $s$, так и времени $u$. Нижний индекс $s$ или $u$ обозначает частную производную по соответствующей переменной.

Используя так называемую аппроксимацию локальной индукции, Да Риос показал [8], что скорость кривой $\mathbf{v}(s, u)$ в каждой точке $s$ вихревой нити (рассматриваемой как подвижная пространственная кривая) дается уравнением локальной индукции

$$
\mathbf{v}=\mathbf{r}_{u}=K \mathbf{b}
$$

Для нерастяжимых кривых использование совместности условий $\mathbf{r}_{u s}=\mathbf{r}_{s u}=\mathbf{t}_{u}=$ $K_{s} \mathbf{b}-K \tau \mathbf{n}$ вместе с подобными условиями совместности для $\mathbf{t}$ и $\mathbf{n}$ приводит к уравнениям [8]

$$
K_{u}=-(K \tau)_{s}-K_{s} \tau, \quad \tau_{u}=\left[\left(\frac{K_{s s}}{K}\right)-\tau^{2}\right]_{s}+K K_{s} .
$$

Эти связанные уравнения для $K$ и $\tau$ были также независимо выведены Бетчовым [9], а потому они называются уравнениями Да Риоса-Бетчова (ДБ). Связь между этими уравнениями кривых и солитонами была обнаружена Хасимото [3], которьй, по сушеству, показал, что используя функиии Хасимото

$$
\psi=K \exp \left[i \int \tau d s\right]
$$

два уравнения (1.3) можно в действительности скомбинировать так, чтобы получить интегрируемое нелинейное уравнение Шредингера (НШ), обладаюшее солитонными решениями,

$$
i q_{u}+q_{s s}+\frac{1}{2}|q|^{2} q=0
$$

при $q=\psi$. Основываясь на этом результате, Лэм предложил [10] общую процедуру, которая позволяет отождествить определенные эволюции пространственных кривых с данным интегрируемым уравнением. Рассматривались такие примеры, как уравнение НШ, уравнение синус-Гордон и модифицированное уравнение Кортевега-де Фриза. Недавно в рамках единого анализа мы показали [11], что в общем случае можно подобным же образом отождествить еще две различные эволюции кривых.

В настояшей работе мы в общих чертах опишем такой анализ, а затем перейдем к уравнению НШ и сконцентрируем внимание на внутренней кинематике трех подвижных 
кривых, связанных с ним. Мы получим связанные эволюционные уравнения для кривизны и кручения каждой из двух новых кривых, являющиеся определенными аналогами уравнений ДБ (1.3). Мы покажем, что каждая из трех подвижных кривых снабжается бесконечным набором геометрических инвариантов, и продемонстрируем их естественную связь с интегрируемым уравнением Ландау-Лифшшица, а для его решений приведем процедуру их использования для построения векторов положений, порождающих все три кривые, связанные с уравнением НШ. В качестве примера мы найдем подвижные кривые для обертываюшей солитонного решения уравнения НШ и сравним их поведение. Интересно, что скорости (в каждой точке) двух новых подвижных кривых, лежащих в основе общей НШ-эволюции, оказьваются определенными нелокальными функциями переменных кривых, совсем не похожими на локальное выражение (уравнение (1.2)) для скорости обычной подвижной кривой, которую до сих пор связьвали с уравнением НШ. Предлагаются возможные применения этих результатов при исследовании движения вихревых нитей в жидкостях.

\section{2. ОТОЖДЕСТВЛЕНИЕ ТРЕХ ЭВОЛЮЦИЙ КРИВЫХ С ДАННЫМ ИНТЕГРИРУЕМЫМ УРАВНЕНИЕМ}

Для описания подвижной кривой удобно ввести [12] следующие уравнения временно́й эволюции для тройки $\Phi$ рене $(\mathbf{t}, \mathbf{n}, \mathbf{b})$ :

$$
\mathbf{t}_{u}=g \mathbf{n}+h \mathbf{b}, \quad \mathbf{n}_{u}=-g \mathbf{t}+\tau_{0} \mathbf{b}, \quad \mathbf{b}_{u}=-h \mathbf{t}-\tau_{0} \mathbf{n}
$$

Параметры $g, h$ и $\tau_{0}$ являются на этом этапе общими параметрами, определяющими временну́ю эволюцию кривой. Они являются функциями как $s$, так и $u$. Нижний индекс $u$ означает частную производную. Налагая условия совместности

$$
\mathbf{t}_{s u}=\mathbf{t}_{u s}, \quad \mathbf{n}_{s u}=\mathbf{n}_{u s}, \quad \mathbf{b}_{s u}=\mathbf{b}_{u s}
$$

и используя уравнения (1.1) и (2.1), получаем

$$
K_{u}=g_{s}-\tau h, \quad \tau_{u}=\left(\tau_{0}\right)_{s}+K h, \quad h_{s}=K \tau_{0}-\tau g
$$

Ниже приведены три формулировки, первая из которых - процедура Лэма [10], а вторая и третья содержат процедуры, приводящие к двум другим различным кривым, которые можно отождествить с заданным интегрируемым уравнением [11]. Для указания на соответствуюшую формулировку мы будем использовать нижние индексы I, II и III для различных параметров кривых, появляющихся в трех формулировках.

ФормУлиРовКА I. Комбинация второго и третьего из уравнений (1.1) немедленно подсказывает определение комплексного вектора $\mathbf{N}=(\mathbf{n}+i \mathbf{b}) \exp \left[i \int \tau d s\right]$ и комплексной функции $\psi$, задаваемой уравнением (1.4). Записывая $\mathbf{N}_{s}, \mathbf{t}_{s}, \mathbf{N}_{u}$ и $\mathbf{t}_{u}$ в терминах $\mathbf{t}$ и 
$\mathbf{N}$, налагая условие совместности $\mathbf{N}_{s u}=\mathbf{N}_{u s}$ и приравнивая в нем коэффишиенты при $\mathbf{t}$ и $\mathbf{N}$, получаем

$$
\psi_{u}+\gamma_{\mathrm{I} s}+\frac{1}{2}\left[\int\left(\gamma_{\mathrm{I}} \psi^{*}-\gamma_{\mathrm{I}}^{*} \psi\right) d s\right] \psi=0,
$$

где

$$
\gamma_{\mathrm{I}}=-(g+i h) \exp \left[i \int \tau d s\right]
$$

Таким образом, при подходящем выборе $\gamma_{\mathrm{I}}$ как функции $\psi$ и ее производных, из $(2.4)$ можно получить известное интегрируемое уравнение на $\psi$. Сравнивая известное решение этого уравнения с функцией Хасимото (1.4), можно найти кривизну $K$ и кручение $\tau$ соответствуюшей подвижной пространственной кривой. Далее, используя указанный

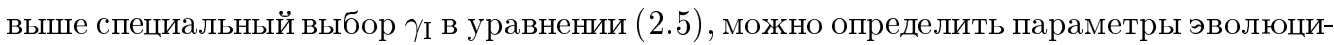
онных кривых $g$ и $h$ как некоторые конкретные функции $K, \tau$ и их производных. Тогда можно также найти $\tau_{0}$ из третьего уравнения в (2.3). Таким образом, явно найден набор параметров кривых $K, \tau, g, h$ и $\tau_{0}$. Другими словами, некоторая подвижная кривая, описываемая этими параметрами, отождествлена таким образом с заданным решением интегрируемого уравнения на $\psi$.

ФоРмУлиРОВКА II. Подходяшим образом комбинируя первые два уравнения в (1.1), видим, что появляются комплексный вектор $\mathbf{M}=(\mathbf{n}-i \mathbf{t}) \exp \left[i \int K d s\right]$ и комплексная функция

$$
\Phi(s, u)=\tau \exp \left[i \int K d s\right] .
$$

Действуя аналогично приведенной выше формулировке І и налагая условие $\mathbf{M}_{s u}=\mathbf{M}_{u s}$, получаем

$$
\Phi_{u}+\gamma_{\mathrm{II} s}+\frac{1}{2}\left[\int\left(\gamma_{\mathrm{II}} \Phi^{*}-\gamma_{\mathrm{II}}^{*} \Phi\right) d s\right] \Phi=0,
$$

где

$$
\gamma_{\mathrm{II}}=-\left(\tau_{0}-i h\right) \exp \left[i \int K d s\right] .
$$

ФоРмУлиРовКА III. Комбинация первого и третьего из уравнений (1.1) приводит к появлению комплексного вектора $\mathbf{P}=(\mathbf{t}-i \mathbf{b})$ и комплексной функции $\chi$, задаваемой как

$$
\chi(s, u)=K+i \tau .
$$

Потребовав $\mathbf{P}_{s u}=\mathbf{P}_{u s}$, получаем

$$
\chi_{u}+\gamma_{\mathrm{III} s}+\frac{1}{2}\left[\int\left(\gamma_{\mathrm{III}} \chi^{*}-\gamma_{\mathrm{III}}^{*} \chi\right) d s\right] \chi=0
$$

где

$$
\gamma_{\mathrm{III}}=-\left(g+i \tau_{0}\right)
$$


Теперь уравнения (2.7) и (2.10) имеют тот же вид, что и уравнение Лэма (2.4). Из обсуждения, следуюшего за формулой (2.5), становится ясно, что при подходяшем выборе

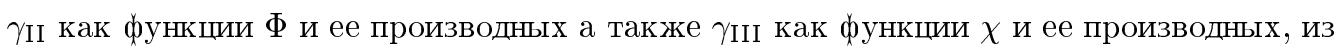
этих уравнений можно получить известные интегрируемые уравнения на $\Phi$ и $\chi$.

Важно отметить, что, как следует из уравнений (1.4), (2.6) и (2.9), комплексные функции $\psi, \Phi$ и $\chi$, удовлетворяюшие (одному и тому же) интегрируемому уравнению в трех формулировках, являются различныцми функциями от $K$ и $\tau$. Более того, мы видим, что комплексные величины $\gamma_{\text {I }}, \gamma_{\text {II }}$ и $\gamma_{\text {III }}$ (см. (2.5), (2.8) и (2.11)), которые возникают в трех формулировках, также включают различные комбинации параметров эволюционных кривых $g, h$ и $\tau_{0}$. Таким образом, ясно, что три формулировки приводят к различным наборам параметров кривых, а потому описывают три различные подвижные пространственные кривые, которые можно связать с данным решением интегрируемого уравнения (заметим, что наш анализ, по-видимому, может быть распространен и на частично-интегрируемые уравнения). В следуюшем разделе, чтобы продемонстрировать это более четко, мы применим эти результаты к уравнению НШ.

\section{3. НЕЛИНЕЙНОЕ УРАВНЕНИЕ ШРЕДИНГЕРА: ДВА АНАЛОГА УРАВНЕНИЙ ДА РИОСА-БЕТЧОВА}

Исходя из трех формулировок, обсуждавшихся в предыдушем разделе, легко проверить, что использование соответствуюшего выбора параметров

$$
\gamma_{\mathrm{I}}=-i \psi_{s}, \quad \gamma_{\mathrm{II}}=-i \Phi_{s}, \quad \gamma_{\mathrm{III}}=-i \chi_{s}
$$

в уравнениях (2.4), (2.7) и (2.10) приводит к уравнению НШ (1.5), причем $q$ отождествляется с тремя комплексными функциями $\psi, \Phi$ и $\chi$, соответственно. Поскольку уравнение НШ является полностью интегрируемым уравнением [2], оно обладает бесконечным набором сохраняюшихся величин $I_{k}, k=1,2, \ldots$, попарно находяшихся в инволюции. Первые три из этих инвариантов даются выражениями ${ }^{1)}$

$$
I_{1}=\int|q|_{s}^{2} d s, \quad I_{2}=\frac{1}{2 i} \int\left(q_{s} q^{*}-q_{s}^{*} q\right) d s, \quad I_{3}=\int\left[\left|q_{s}\right|^{2}-\frac{1}{4}|q|^{4}\right] d s, \ldots
$$

Рассмотрим эти инварианты в каждой из трех формулировок.

\footnotetext{
1) 3 десь $I_{3}$ - гамильтониан, который приводит к уравнению (1.5) при использовании скобок Пуассона$$
\{F, G\}=i \int\left[\frac{\delta F}{\delta q} \frac{\delta G}{\delta q^{*}}-\frac{\delta G}{\delta q} \frac{\delta F}{\delta q^{*}}\right] d s
$$ 
I. Полагая $q=\psi$ в (1.5) и приравнивая вешественные и мнимые части, получаем уравнения ДБ (1.3). Следовательно, они также обладают бесконечным числом сохраняюшихся величин, получаемых при подстановке $q=\psi$ в (3.2). Инварианты теперь появляются как геометрические связи [13]:

$$
I_{1}=\int K^{2} d s, \quad I_{2}=\int K^{2} \tau d s, \quad I_{3}=\int\left[K_{s}^{2}+K^{2} \tau^{2}-\frac{1}{4} K^{4}\right] d s, \ldots
$$

Далее, внимательное рассмотрение уравнений (1.3) показывает, что полное кручение $I_{0}=\int \tau d s$ также сохраняется. У этой дополнительной геометрической связи нет аналога среди инвариантов (3.2) уравнения НШ.

II. В этом случае мы полагаем $q=\Phi$ в (1.5) и получаем связанные уравнения

$$
K_{u}=\left[\left(\frac{\tau_{s s}}{\tau}\right)-K^{2}\right]_{s}+\tau \tau_{s}, \quad \tau_{u}=-(K \tau)_{s}-\tau_{s} K
$$

Это дает первый аналог уравнений ДБ (1.3). Из сравнения $\psi$ из (1.4) и $\Phi$ из (2.6) очевидно, что этот аналог можно найти просто путем взаимной замены $K$ и $\tau$ в (1.3). Таким образом, соответствуюшее бесконечное число геометрических связей также можно найти, используя эту замену в (3.3):

$$
I_{1}=\int \tau^{2} d s, \quad I_{2}=\int \tau^{2} K d s, \quad I_{3}=\int\left[\tau_{s}^{2}+\tau^{2} K^{2}-\frac{1}{4} \tau^{4}\right] d s, \ldots
$$

Полная кривизна $I_{0}=\int K d s$ также сохраняется, что не имеет аналога среди сохраняющихся плотностей (3.2) уравнения НШ.

III. Наконец, полагая $q=\chi$ в (1.5), получаем следующий второй аналог уравнений ДБ:

$$
K_{u}=-\tau_{s s}-\frac{1}{2}\left(K^{2}+\tau^{2}\right) \tau, \quad \tau_{u}=K_{s s}+\frac{1}{2}\left(K^{2}+\tau^{2}\right) K
$$

Далее, полагая $q=\chi$ в (3.2), получаем третий бесконечный набор геометрических связей:

$$
\begin{gathered}
I_{1}=\int\left(K^{2}+\tau^{2}\right) d s, \quad I_{2}=\int\left(K_{s} \tau-K \tau_{s}\right) d s, \\
I_{3}=\int\left[K_{s}^{2}+\tau_{s}^{2}-\frac{1}{4}\left(K^{2}+\tau^{2}\right)^{2}\right] d s, \ldots .
\end{gathered}
$$

В заключение этого раздела заметим, что полная длина кривой $L=\int d s$ также сохраняется во всех трех формулировках, поскольку кривые являются нерастяжимыми. 


\section{4. ПОСТРОЕНИЕ ТРЕХ КРИВЫХ С ИСПОЛЬЗОВАНИЕМ УРАВНЕНИЯ ЛАНДАУ-ЛИФШИЦА}

Общее решение уравнения НШ (1.5) имеет вид $q=\rho e^{i \theta}$. Приравнивая это к трем комплексным функциям, определенным формулами (1.4), (2.6) и (2.9), получаем кривизну и кручение трех пространственных кривых в виде $\kappa_{\mathrm{I}}=\rho, \tau_{\mathrm{I}}=\theta_{s}(\mathrm{I}), \kappa_{\mathrm{II}}=\theta_{s}, \tau_{\mathrm{II}}=\rho(\mathrm{II})$ и $\kappa_{\text {III }}=\rho \cos \theta, \tau_{\text {III }}=\rho \sin \theta$ (III). Ясно, что это три различные пространственные кривые, каждая из которых имеет известные кривизну и кручение. Однако их использование для явного решения уравнений Френе-Серре (1.1) для $\mathbf{t}$ и дальнейшего построения вектора положения $\mathbf{r}(s, u)=\int \mathbf{t} d s$ (эволюционирующей) пространственной кривой на практике оказывается нетривиальной задачей. Здесь мы покажем, что имеется определенная связь между тремя эволюционными кривыми и интегрируемым уравнением Ландау-Лифшшица (ЛЛ) [14], что дает альтернативную процедуру построения трех подвижных кривых.

Сначала приравняем выражения для $\gamma_{\mathrm{I}}, \gamma_{\mathrm{II}}$ и $\gamma_{\text {III }}(3.1)$ к выражениям $(2.5),(2.8)$ и (2.11). Это дает следуюшие параметры эволюции кривых $g, h$ и $\tau_{0}$ в трех случаях:

$$
\begin{array}{llll}
\mathrm{I}: & g_{\mathrm{I}}=-\kappa_{\mathrm{I}} \tau_{\mathrm{I}}, & h_{\mathrm{I}}=\kappa_{\mathrm{I} s}, & \tau_{0 \mathrm{I}}=\frac{\kappa_{\mathrm{IIs} s}}{\kappa_{\mathrm{I}}}-\tau_{\mathrm{I}}^{2} ; \\
\mathrm{II}: & g_{\mathrm{II}}=\frac{\tau_{\mathrm{II} s} s}{\tau_{\mathrm{II}}}-\kappa_{\mathrm{II}}^{2}, & h_{\mathrm{II}}=-\tau_{\mathrm{II} s}, & \tau_{\mathrm{OII}}=-\kappa_{\mathrm{II}} \tau_{\mathrm{II}} ; \\
\mathrm{III}: & g_{\mathrm{III}}=-\tau_{\mathrm{III} s}, & h_{\mathrm{III}}=\frac{1}{2}\left(\kappa_{\mathrm{III}}^{2}+\tau_{\mathrm{III}}^{2}\right), & \tau_{0 \mathrm{III}}=\kappa_{\mathrm{III} s} .
\end{array}
$$

Соответствуюшим образом подставляя эти выражения для каждой из формулировок в уравнения (2.1) и используя уравнения (1.1), с помошью простого вычисления [11] можно показать, что в каждом случае получается полностью интегрируемое уравнение ЛЛ [14]

$$
\mathbf{S}_{u}=\mathbf{S} \times \mathbf{S}_{s s}, \quad \mathbf{S}^{2}=1,
$$

т.е. уравнение (4.4) удовлетворяется касательной $\mathbf{t}_{\mathrm{I}}$ к подвижной пространственной

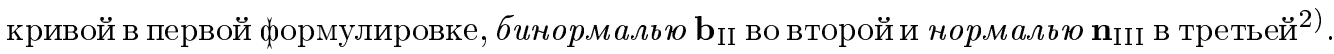
Первый из вьшеприведенных случаев можно рассматривать как обратное отображение Лакшманана [4], при этом, исходя из уравнения ЛЛ и отож дествляя $\mathbf{S}$ с касательной к подвижной кривой, получаем уравнения ДБ, а из них - уравнение НШ для $\psi$. Обратные к двум другим отображения дают два аналога уравнений ДБ, полученных в разделе 3 , и, очевидно, приводят к новым геометриям, связанным с уравнением НШ.

Было показано, что уравнение ЛЛ (4.4) является полностью интегрируемым [15] и калибровочно-эквивалентным уравнению НШ [16]. Его точные решения можно найти

\footnotetext{
2) Гамильтонианы для этих трех эволюций представляют собой просто соответствующие инварианты $I_{1}$, заданные в $(3.3),(3.5)$ и (3.7). Их можно записать в виде $H=\int\left(\mathbf{S}_{s}\right)^{2} d s$, где вектор спина $\mathbf{S}$ отождествляется с $\mathbf{t}_{\mathrm{I}}, \mathbf{b}_{\text {II }}$ и $\mathbf{n}_{\text {III }}$, соответственно. Таким образом, компоненты каждого из этих трех векторов удовлетворяют обычным скобкам Пуассона для момента импульса.
} 
в работах [15], [17]. Теперь мы в общих чертах наметим [18], как векторы положений $\mathbf{r}_{\mathrm{I}}, \mathbf{r}_{\text {II }}$ и $\mathbf{r}_{\text {III }}$, порождающие три подвижные кривые, лежашие в основе уравнения НШ, можно найти в терминах точных решений $\mathbf{S}$ уравнения (4.4). Пусть $\left(\mathbf{t}_{i}, \mathbf{n}_{i}, \mathbf{b}_{i}\right), i=\mathrm{I}$, II и III, обозначают тройки Френе трех подвижных кривых, удовлетворяющих уравнениям (1.1), с соответствуюшими параметрами $\kappa_{i}$ и $\tau_{i}$.

I. В этом случае $\mathbf{t}_{\mathrm{I}}=\mathbf{r}_{\mathrm{I} s}$ является касательной к некоторой подвижной кривой, созданной вектором положения $\mathbf{r}_{\mathrm{I}}(s, u)$. Пусть $\mathbf{t}_{\mathrm{I}}=\mathbf{S}$ - известное решение уравнения (4.4). Тогда

$$
\kappa_{\mathrm{I}}=\left|\mathbf{t}_{\mathrm{I} s}\right|=\left|\mathbf{S}_{s}\right|, \quad \tau_{\mathrm{I}}=\frac{\mathbf{t}_{\mathrm{I}}\left(\mathbf{t}_{\mathrm{I} s} \times \mathbf{t}_{\mathrm{I} s s}\right)}{\mathbf{t}_{\mathrm{I} s}^{2}}=\frac{\mathbf{S}\left(\mathbf{S}_{s} \times \mathbf{S}_{s s}\right)}{\mathbf{S}_{s}^{2}}
$$

а поэтому соответствующая подвижная кривая $\mathbf{r}_{\mathrm{I}}(s, u)$ дается уравнением

$$
\mathbf{r}_{\mathrm{I}}(s, u)=\int \mathbf{t}_{\mathrm{I}} d s=\int \mathbf{S}(s, u) d s
$$

Это выражение для $\mathbf{r}_{\text {I }}$ совпадает с выражением для поверхности, которое можно получить, используя метод солитонных поверхностей Сима [19].

II. В этом случае $\mathbf{b}_{\text {II }}$ является бинормалью к некоторой подвижной кривой $\mathbf{r}_{\mathrm{II}}(s, u)$. Положим $\mathbf{b}_{\mathrm{II}}=\mathbf{S}$. Имеем $\mathbf{t}_{\mathrm{II}}=\mathbf{r}_{\mathrm{II} s}$. Кривизна $\kappa_{\mathrm{II}}=\tau_{\mathrm{I}}$ и кручение $\tau_{\mathrm{II}}=\kappa_{\mathrm{I}}($ cм. $(4.5))$. Можно показать, что порождаюший подвижную кривую вектор положения $\mathbf{r}_{\text {II }}(s, u)$ имеет вид [18]

$$
\mathbf{r}_{\mathrm{II}}(s, u)=\int \mathbf{t}_{\mathrm{II}} d s=\int \frac{\mathbf{S}}{\kappa_{\mathrm{I}}} \times \mathbf{S}_{s} d s .
$$

III. В данном случае $\mathbf{n}_{\text {III }}$ является нормалью к некоторой другой подвижной кривой $\mathbf{r}_{\mathrm{III}}(s, u)$. Положим поэтому $\mathbf{n}_{\mathrm{III}}=\mathbf{S}$. Далее, $\mathbf{t}_{\mathrm{III}}=\mathbf{r}_{\mathrm{III} s}$. Из уравнений Френе-Серpe (1.1) для этой тройки получаем

$$
\left(\kappa_{\text {III }}^{2}+\tau_{\text {III }}^{2}\right) \mathbf{t}_{\text {III }}=\tau_{\text {III }}\left(\mathbf{n}_{\text {III }} \times \mathbf{n}_{\text {III } s}\right)-\kappa_{\text {III }} \mathbf{n}_{\text {III } s}
$$

Простое вычисление показывает [18], что $\kappa_{\text {III }}=\kappa_{\mathrm{I}} \cos \eta_{\mathrm{I}}$ и $\tau_{\mathrm{III}}=\kappa_{\mathrm{I}} \sin \eta_{\mathrm{I}}$, где $\eta_{\mathrm{I}}=$ $\int \tau_{\mathrm{I}} d s+c_{\mathrm{I}}(u)$. Здесь $c_{\mathrm{I}}(u)-$ функция времени $u$, которую можно определить через $\kappa_{\mathrm{I}}$ и $\tau_{\text {I }}$ с помощью простого выгисления, использующего уравнения на $\kappa_{\text {III } u}$ и $\tau_{\text {III } u}$, полу-

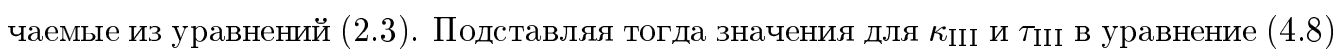
и полагая $\mathbf{n}_{\mathrm{III}}=\mathbf{S}$, можно найти вектор положения $\mathbf{r}_{\mathrm{III}}(s, u)$, порождающий третью подвижную пространственную кривую [18]:

$$
\mathbf{r}_{\mathrm{III}}(s, u)=\int \mathbf{t}_{\mathrm{III}} d s=\int \frac{\left(\mathbf{S} \times \mathbf{S}_{s}\right) \sin \eta_{\mathrm{I}}-\mathbf{S}_{s} \cos \eta_{\mathrm{I}}}{\kappa_{\mathrm{I}}} d s
$$




\section{5. ПОДВИЖНЫЕ ПРОСТРАНСТВЕННЫЕ КРИВЫЕ, СВЯЗАННЫЕ С НШ-СОЛИТОНОМ}

Как было показано в предыдушем разделе, выражения для трех подвижных кривых, связанных с уравнением НШ, определяются соответственно формулами (4.6), (4.7) и (4.9) через известное решение $\mathbf{S}$ уравнения ЛЛ (4.4). Определяя три ортогональных единичных вектора $\hat{\mathbf{e}}_{1}=\{1,0,0\}, \hat{\mathbf{e}}_{2}=\{0, \cos \eta, \sin \eta\}, \hat{\mathbf{e}}_{3}=\{0,-\sin \eta, \cos \eta\}$, можно записать солитонное решение [17] уравнения (4.4) в виде

$$
\mathbf{S}(s, u)=\left(1-\mu \nu \operatorname{sech}^{2}(\nu \xi)\right) \hat{\mathbf{e}}_{1}+\mu \nu \operatorname{sech}(\nu \xi) \operatorname{th}(\nu \xi) \hat{\mathbf{e}}_{2}-\mu \lambda \operatorname{sech}(\nu \xi) \hat{\mathbf{e}}_{3},
$$

где $\xi=s-2 \lambda u, \eta=\lambda s+\left(\nu^{2}-\lambda^{2}\right) u$ и $\mu=2 \nu /\left(\nu^{2}+\lambda^{2}\right), \nu$ и $\lambda$ - произвольные константы. Как ясно из нашего обсуждения в разделе 4 , три кривые, получаюшиеся из этого решения, связаны с солитонным решением $q=2 \nu \operatorname{sech}(\nu \xi) e^{i \eta}$ уравнения НШ (1.5). Их можно найти следуюшим образом.

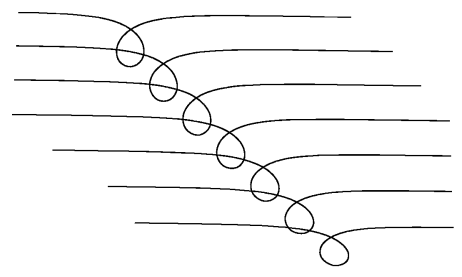

Рис. 1. Стробоскопический график эволюционирующей пространственной кривой $\mathbf{r}_{\mathrm{I}}(s, u)(5.2)$ при $\nu=1$ и $\lambda=0.1$.

I. Подставляя (5.1) в $(4.5)$, получаем $\kappa_{\mathrm{I}}=2 \nu \operatorname{sech}(\nu \xi)$ и $\tau_{\mathrm{I}}=\lambda$. Далее, подстановка (5.1) в (4.6) дает

$$
\mathbf{r}_{\mathrm{I}}=(s-\mu \operatorname{th}(\nu \xi),-\mu \operatorname{sech}(\nu \xi) \cos \eta,-\mu \operatorname{sech}(\nu \xi) \sin \eta) .
$$

Видно, что это согласуется с результатом, полученным в работе [20] с использованием процедуры Сима [19]. На рис. 1 представлен стробоскопический график подвижной кривой (5.2) в различные моменты времени. Он описьвает распространение хорошо известного "петлевого" солитона Хасимото. Между моментами времени, при которых мы изобразили кривую, петля изменяет свой размер и, кроме того, врашается вокруг асимптотического направления, которое данная кривая принимает при $s \rightarrow \pm \infty$. На рисунке мы для простоты не приводим эти промежуточные моменты времени. Как видно из рисунка, петля восстанавливает свою форму в последуюшие моменты времени.

II. В этом случае получаем $\kappa_{\mathrm{II}}=\lambda$ и $\tau_{\mathrm{II}}=2 \nu \operatorname{sech}(\nu \xi)$. Подставляя (5.1) в (4.7), находим подвижную кривую в виде

$$
\mathbf{r}_{I I}=\int\left[\mu \lambda \operatorname{sech}(\nu \xi) \hat{\mathbf{e}}_{1}-\mu \lambda \operatorname{th}(\nu \xi) \hat{\mathbf{e}}_{2}+\frac{\left(\lambda^{2}-\nu^{2}\right)}{\left(\lambda^{2}+\nu^{2}\right)} \hat{\mathbf{e}}_{3}\right] d s .
$$


При этом, поскольку кривизна является постоянной и кручение обращается в нуль при $s \rightarrow \pm \infty$ при всех конечных моментах времени, кривая ограничена двумя планарными окружностями на обоих концах. Максимум непланарности кривой достигается при $s=$ $2 \lambda u$. Стробоскопический график кривой (5.3) приведен на рис. 2. Ясно видно, что эта кривая врашается и распространяется с течением времени.

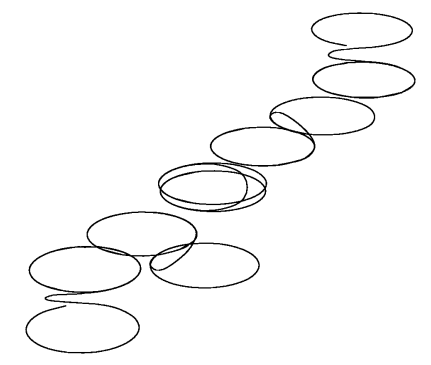

Рис. 2. То же, что на рис. 1 , но для кривой $\mathbf{r}_{\mathrm{II}}(s, u)(5.3)$ при $\nu=0.3$ и $\lambda=0.1$.

III. В этом случае $\kappa_{\text {III }}=2 \nu \operatorname{sech}(\nu \xi) \cos \eta$ и $\tau_{\text {III }}=2 \nu \operatorname{sech}(\nu \xi) \sin \eta$. После подстановки (5.1) в (4.9) при $\eta_{\mathrm{I}}=\eta$ в результате длинного, но несложного вычисления получаем

$$
\begin{aligned}
\mathbf{r}_{\mathrm{III}}= & \int\left[\mu \operatorname{sech}(\nu \xi)(\lambda \sin \eta-\nu \operatorname{th}(\nu \xi) \cos \eta) \hat{\mathbf{e}}_{1}-\right. \\
& -\left(\mu \lambda \operatorname{th}(\nu \xi) \sin \eta+\left(1-\mu \nu \operatorname{th}^{2}(\nu \xi)\right) \cos \eta\right) \hat{\mathbf{e}}_{2}+ \\
& \left.+\left(\frac{\lambda^{2}-\nu^{2}}{\lambda^{2}+\nu^{2}} \sin \eta-\mu \lambda \operatorname{th}(\nu \xi) \cos \eta\right) \hat{\mathbf{e}}_{3}\right] d s
\end{aligned}
$$

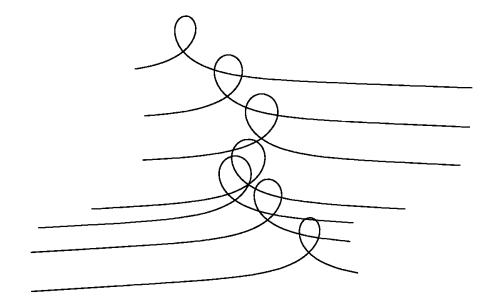

Рис. 3. То же, что на рис. 1 , но для кривой $\mathbf{r}_{\mathrm{III}}(s, u)(5.4)$ при $\nu=1$ и $\lambda=0.3$.

Стробоскопический график подвижной кривой (5.4) в различные моменты времени приведен на рис. 3 , где изображено распространение “петлевого" солитона, отличного от солитона Хасимото в том смысле, что он осциллирует во времени. Более того, при промежуточных временах петля врашается вокруг асимптотического направления, что уменьшает размер петли, и периодически почти выпрямляется, после чего снова начинается закручивание в петлю. Эти промежуточные графики не представлены на рисунке. 


\section{6. СКОРОСТИ ТРЕХ КРИВЫХ, СВЯЗАННЫХ С ЭВОЛЮЦИЕЙ НЕЛИНЕЙНОГО УРАВНЕНИЙ ШРЕДИНГЕРА}

Возвращаясь к общим результатам из раздела 4, напомним, что три вектора положений $\mathbf{r}_{i}, i=\mathrm{I}$, II и III, которые связаны с эволюцией уравнения НШ, можно найти соответственно из соотношений (4.6), (4.7) и (4.9), используя точное решение $\mathbf{S}$ уравнения ЛЛ (4.4). Соответствуюшие скорости кривых $\mathbf{v}_{i}(s, u)=\mathbf{r}_{i u}$ в каждой точке $s$ можно поэтому найти из этих уравнений путем прямого дифференцирования по времени $u$. Однако чтобы сравнить и противопоставить внутренние геометрии трех пространственных кривых, поучительно выразить эти скорости в терминах векторов соответствующих троек Френе так же, как и параметры кривых, следующим образом. Поскольку кривые нерастяжимы, имеем $\mathbf{v}_{i s}=\mathbf{r}_{i u s}=\mathbf{r}_{i s u}=\mathbf{t}_{i u}$. С другой стороны, из первого уравнения в (2.1) получаем $\mathbf{t}_{i u}=g_{i} \mathbf{n}_{i}+h_{i} \mathbf{b}_{i}$. Здесь величины $g_{i}$ и $h_{i}$ для трех кривых, связанных с уравнением НШ, задаются соответственно уравнениями (4.1), (4.2) и (4.3). Используя это в выражении

$$
\mathbf{v}_{i}(s, u)=\int \mathbf{t}_{i u} d s
$$

получаем

$$
\begin{array}{ll}
\text { I : } & \mathbf{v}_{\mathrm{I}}=\int\left(-\kappa_{\mathrm{I}} \tau_{\mathrm{I}} \mathbf{n}_{\mathrm{I}}+\kappa_{\mathrm{I} s} \mathbf{b}_{\mathrm{I}}\right) d s=\kappa_{\mathrm{I}} \mathbf{b}_{\mathrm{I}}, \\
\mathrm{II}: & \mathbf{v}_{\mathrm{II}}=\int\left[\left(\frac{\tau_{\mathrm{II} s s}}{\tau_{\mathrm{II}}}-\kappa_{\mathrm{II}}^{2}\right) \mathbf{n}_{\mathrm{II}}-\kappa_{\mathrm{II} s} \mathbf{b}_{\mathrm{II}}\right] d s, \\
\mathrm{III}: & \mathbf{v}_{\mathrm{III}}=\int\left[-\tau_{\mathrm{III} s} \mathbf{n}_{\mathrm{III}}+\frac{1}{2}\left(\kappa_{\mathrm{III}}^{2}+\tau_{\mathrm{III}}^{2}\right) \mathbf{b}_{\mathrm{III}}\right] d s .
\end{array}
$$

Как и ожидалось, $\mathbf{v}_{\text {I }}$ совпадает со скоростью вихревой нити (1.2), выведенной Да Риосом в механике жидкости. Это выражение локально по переменным кривых: скорость

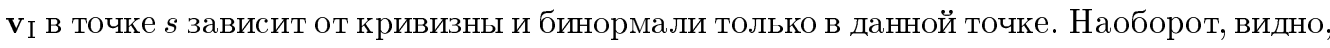

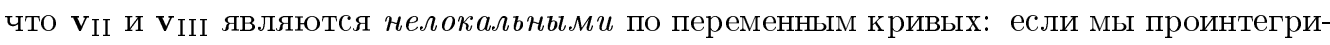
руем по частям правые части уравнений (6.2) и (6.3) и многократно применим уравнения Френе-Серре (1.1) в получаюшемся выражении, то обе эти скорости примут вид $\mathbf{v}_{i}=A_{i} \mathbf{t}_{i}+B_{i} \mathbf{n}_{i}+C_{i} \mathbf{b}_{i}, i=\mathrm{II}, \mathrm{III}$, где компоненты $A_{i}, B_{i}$ и $C_{i}$ можно записать в терминах интегралов некоторых функций, включаюших кривизну, кручение, их высшие производные и их различные произведения. Интересно, что несмотря на такое сложное поведение этих скоростей, соответствующие им эволюции кривых обладают бесконечным числом констант движения. Это, по существу, вытекает из их связи с уравнением Нш. А именно, это имеет место потому, что, как мы показали в разделе 3 , бинормаль $\mathbf{b}_{\text {II }}$ второй кривой и нормаль $\mathbf{n}_{\text {III }}$ третьей кривой удовлетворяют интегрируемому уравнению ЛЛ.

В заключение сделаем следующие замечания. В жидкости, как хорошо известно, индуцированная скорость $\mathbf{v}$ в точке определяется с помощью формулы Био-Савара как 
объемный интеграл, включающий напряженность вихря $\boldsymbol{\Omega}$. Следует отметить, во-первых, что если в этой формуле выразить $\Omega$ через векторы тройки $\Phi$ рене для нити, то в любой реалистичной модели жидкости скорость $\mathbf{v}$ является нелокальной по переменным кривой и становится локальной только в рамках некоторой аппроксимации. Во-вторых, в интересном эксперименте с жидкостью во врашающемся баке Хопфингер и Брауэнд наблюдали [21] компактные искажения, которые врашаются и распространяются вдоль ядра вихря как солитон. Это означает, что наши новые результаты о связи скоростей $\mathbf{v}_{\text {II }}$ и $\mathbf{v}_{\text {III }}$ с уравнением НШ и его солитонами могут иметь значение для реальных жидкостей. Ввиду этого интерес могут представлять соответствующее теоретическое моделирование завихренности, а также возможные экспериментальные исследования деталей геометрической структуры подвижных вихревых нитей в поисках какого-либо сходства с изображенным на рис. 1-3 распространением компактных искажений.

\section{Список литературы}

[1] D. J. Struik. Lectures on Classical Differential Geometry. Reading, MA: Addison-Wesley, 1961.

[2] M. J. Ablowitz, H. Segur. Solitons and the Inverse Scattering Transform. Philadelphia: SIAM, 1981.

[3] H. Hasimoto. J. Fluid Mech. 1972. V. 51. P. 477

[4] M. Lakshmanan. Phys. Lett. A. 1977. V. 61. P. 53; R. Balakrishnan. J. Phys. C. 1982. V. 15. P. L1305.

[5] J.P. Keener. Physica D. 1998. V. 31. P. 269.

[6] K. W. Schwarz. Phys. Rev. B. 1998. V. 38. P. 2398.

[7] R. E. Goldstein, D. M. Petrich. Phys. Rev. Lett. 1991. V. 67. P. 3203.

[8] L. S. Da Rios. Rend. Circ. Mat. Palermo. 1906. V. 22. P. 117.

[9] R. Betchov. J. Fluid Mech. 1965. V. 22. P. 471; R. L. Ricca. Nature. 1991. V. 352. P. 561.

[10] G. L. Lamb. J. Math. Phys. 1977. V. 18. P. 1654.

[11] S. Murugesh, R. Balakrishnan. Phys. Lett. A. 2001. V. 290. P. 81; nlin.PS/0104066.

[12] R. Balakrishnan, A.R. Bishop, R. Dandoloff. Phys. Rev. B. 1993. V. 47. P. 3108; Phys. Rev. Lett. 1990. V. 64. P. 2107; R. Balakrishnan, R. Blumenfeld. J. Math. Phys. 1997. V. 38. P. 5878.

[13] J. Langer, R. Perline. J. Math. Phys. 1993. V. 35. P. 1732.

[14] L. D. Landau, E. M. Lifshitz. Phys. Z. Sow. 1935. V. 8. P. 153.

[15] L. A. Takhtajan. Phys. Lett. A. 1977. V. 64. P. 235.

[16] В. Е. Захаров, Л. А. Тахтаджсян. ТМФ. 1979. Т. 38. № 1. С. 26.

[17] J. Tjon, J. Wright. Phys. Rev. B. 1977. V. 15. P. 3470.

[18] S. Murugesh, R. Balakrishnan. Eur. Phys. J. B. 2002. V. 29. P. 193; nlin.PS/0111052.

[19] A. Sym. Lett. Nuovo Cimento. 1978. V. 22. P. 420.

[20] D. Levi, A. Sym, S. Wojciechowski. Phys. Lett. A. 1983. V. 94. P. 408.

[21] E. J. Hopfinger, F. K. Browand. Nature. 1982. V. 295. P. 393. 